\title{
Téoros
}

Revue de recherche en tourisme

\section{Coûts et prix bas}

\section{Vers une logique de marketing stratégique}

\section{Philippe Callot}

Volume 22, numéro 3, automne 2003

L’accessibilité, une conquête inachevée

URI : https://id.erudit.org/iderudit/1071346ar

DOI : https://doi.org/10.7202/1071346ar

Aller au sommaire du numéro

Éditeur(s)

Université du Québec à Montréal

ISSN

0712-8657 (imprimé)

1923-2705 (numérique)

Découvrir la revue

\section{Citer cet article}

Callot, P. (2003). Coûts et prix bas : vers une logique de marketing stratégique. Téoros, 22(3), 37-41. https://doi.org/10.7202/1071346ar d'utilisation que vous pouvez consulter en ligne.

https://apropos.erudit.org/fr/usagers/politique-dutilisation/ 


\section{Coûts et prix bas}

\section{Vers une logique de marketing stratégique}

\section{Philippe Callot}

$\mathbf{T}$ outes les entreprises n'ont pas les mêmes structures de coûts. Toutes ne s'adressent pas aux mêmes marchés. Ces évidences méritent d'être rappelées car, dans une conjoncture dite difficile où la concurrence est agressive et l'environnement turbulent, le prix se réaffirme comme un élément clé du positionnement de la firme. La concurrence est définitivement globale. Dans un tel contexte, la concurrence des firmes s'aiguise au fil des expériences, des apprentissages, des comparaisons (benchmark), des échecs... La variable du prix et son importance au cœur du marketing-mix non démentie depuis longtemps (Kotler, 1971) jouent un rôle majeur dans la stratégie marketing des opérateurs concurrents (Balfet, 2001 : ch. 5.3). Les évolutions conceptuelles autour de cette variable du marketing-mix, entre élasticité, yield management, target costing, tarification différenciée, indice de popularité par exemple, montrent que rien n'est acquis et que tout peut être remis en cause sous l'impulsion de variables contingentes et en s'appuyant sur des concepts anciens ou émergents.

Comme la variable du produit (et du service) évolue au rythme des croissances, des besoins et des souhaits du consommateur, celle du prix s'affiche comme la clé d'une conquête inachevée pour de nouveaux marchés, de nouveaux segments, de nouvelles clientèles. L'évolution du confort moyen dans les économies développées, l'évolution du comportement du consommateur (plus exigeant, « zappeur », mieux formé, plus attentif), l'évo- lution des procédés (technologies, maîtrise des processus, meilleure productivité, création de valeur maitrisée) rendent la concurrence sans cesse renouvelée et donc cette conquête du consommateur inachevée (Callot, 1997). Les positions affirmées comme stables hier sont un leurre.

Les secteurs évoluent au rythme de la contingence environnementale. La variable du prix, dynamique par l'approche de nouvelles demandes ou de coûts toujours mieux maîtrisés, aide ou favorise les entreprises à saisir les occasions marchandes ou la révision de positions supposées établies. Dans cette configuration globale et contingente, cette approche du prix renvoie à son optimisation selon la nature de l'activité, la période, la structure des coûts de chaque firme. " La définition d'un niveau 'optimal' de prix suppose une connaissance préalable des objectifs et des moyens de l'entreprise mais aussi de son environnement concurrentiel » (Mahé de Boislandelle, 1998).

Le présent article met l'accent sur l'effet des bas prix au sein de secteurs très concurrentiels à partir de modèles porteriens. Ces trois secteurs sont : le transport aérien, la restauration commerciale et l'hôtellerie économique.

\section{Les trois secteurs en rapport avec les deux modèles}

Les compagnies EasyJet et Ryanair se montrent les plus agressives dans le secteur aérien, dans un marché qui s'est révélé comme dynamique et générateur de profits ${ }^{2}$

De quoi s'agit-il ? Pour commenter leur réussite ${ }^{3}$ et tenter de "décortiquer »le succès de ce phénomène des bas prix, nous nous appuierons sur le cas d'EasyJet dans le domaine aérien et, notamment, sur la définition de son business model, ainsi que sur le St. Clair, établissement situé à Angers, qui œuvre dans le domaine de la restauration commerciale, PME d'un secteur lui aussi turbulent. Les établissements familiaux où le débit est assuré (taux de fidélité élevé et donc un nombre de repas qui ne varie pas beaucoup d'un jour à l'autre) sont, en restauration commerciale, des lieux à (re)fréquenter.

Nous évoquerons aussi l'hôtellerie économique (Formule 1, B\&B, Première Classe, pour ne citer que ces enseignes). Nous comparerons leurs pratiques managériales aux deux modèles de Michael E. Porter, professeur à l'université Harvard :

- les trois stratégies de base (Porter, 1982);

- la chaîne de valeur (Porter, 1985 ; 1993).

Pour certains, ils paraîtront surannés. Pour autant, nous avons pu constater, à l'émergence de ces nouveaux entrants du secteur aérien et à la disparition d'autres opérateurs (Swissair par exemple et Swiss actuellement, les pertes d'Alitalia de 907 millions d'euros en 2001 ou encore plus récemment la catastrophe sociale de la liquidation d'Air Lib et ses répercussions sur 3200 salariés directs et 18000 indirects), que ces modèles n'avaient pas pris une ride et s'imposaient de façon criante en termes stratégiques.

\section{Le premier modèle : les trois stratégies de base}

Au-delà de la technique du yield management largement décrite désormais (Dubois et al, 1995 ; Capiez et al., 
2002), nous tenterons de montrer que les succès de ces différentes firmes sont le parfait reflet de modèles stratégiques énoncés et adaptés dans un contexte donné, ici ceux du domaine aérien, de l'hôtellerie économique et de la restauration commerciale à petits prix. Selon un des dirigeants d'EasyJet, le modèle d'affaires d'EasyJet s'articule autour de trois thèmes qui se rapprochent très fortement des préconisations de Porter et de ses trois stratégies de base.

Quelle est la stratégie des compagnies « à bas coûts/prix » ?

- EasyJet est une compagnie aérienne régulière opérant des vols de point à point (sans connections d'une longueur maximale de 2 h 30) [...] ;

- La simplification de la tarification est la clé du système à savoir un management des prix du bas vers le haut soit l'inverse de ce qui se fait généralement dans le marché traditionnel [...] ;

- La maîtrise essentielle des coûts en optimisant le matériel et le personnel (Thevenaz ${ }^{4}, 2001$ ).

Ces trois orientations sont on ne peut plus proches des définitions des trois stratégies de base spécifiées dans l'ouvrage de référence : Choix stratégiques et concurrence (Porter, 1982) et résumées à la figure 1 .

Les deux autres stratégies de base sont au cœur de la logique du phénomène des bas prix (domination par les coûts), toujours au sens de Porter.
Cette logique se trouve dans la suite de la définition de la stratégie de concentration. «Il en résulte que la firme parvient soit à une différenciation, parce qu'elle satisfait mieux les besoins de la cible particulière, soit à des coûts plus faibles pour desservir cette cible, soit aux deux à la fois »(Porter, 1982 : 43). Nous pouvons comparer ce modèle au choix et au positionnement stratégique des trois exemples d'entreprises.

\section{Les compagnies aériennes}

Concentration, différenciation et domination en matière de coûts sont les adaptations praticiennes réalisées par la compagnie low cost. En se spécialisant dans les vols d'une longueur maximale de 2 h 30 sur un marché « oligopolistique et contesté », EasyJet s'inscrit parfaitement dans la logique de concentration émise par Porter 5

Dans ce marché en explosion, les créations de connexions sont nombreuses et attestent de cette orientation de concentration : Londres-Tours ou Londres-Reims pour Ryanair ou encore Genève-Liverpool pour EasyJet sont des dessertes «nouvelles » qui crédibilisent l'action de concentration.

Créée en avril 2002, la ligne LondresTours a séduit d'emblée une clientèle anglaise (80\% des utilisateurs sont issus du Royaume-Uni). Le gain de temps (37\% des motifs d'utilisation de cette desserte), les prix (33\%) et le côté « pratique » $(22 \%)$ sont les déterminants majeurs d'utilisation que les clients ont identifiés dans d'une enquête estivale ${ }^{6}$.

\section{FIGURE 1}

Les trois stratégies de base selon Porter (1982)

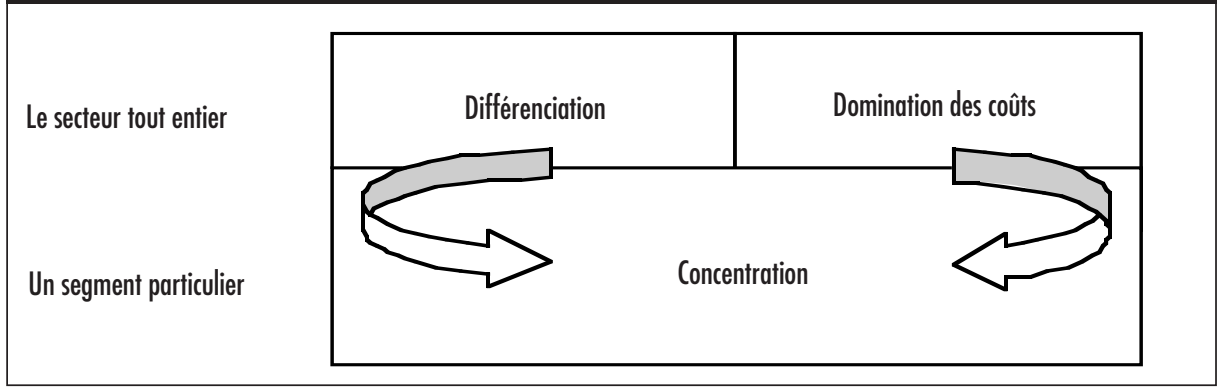

La concentration de l'activité consiste à se concentrer sur un groupe de clients particulier, sur un segment de la gamme des produits, sur un marché géographique [...]. Cette stratégie repose sur l'idée que la firme est capable de desservir sa cible stratégique restreinte plus efficacement, ou en consommant moins de ressources que les concurrents qui luttent dans un domaine plus large, (Porter, 1982 : 42)
La domination en matière de coûts s'exprime par la volonté d'efficience des opérateurs. La maintenance n'est pas réduite (comme certaines rumeurs le font croire), mais optimisée du fait qu'un seul type d'avion était, à ce jour ${ }^{7}$, utilisé par exemple chez EasyJet (Boeing 737).

La réduction des services à bord et la réduction des temps d'attente au sol sont également d'autres éléments qui contribuent à augmenter la valeur interne de la firme (deuxième modèle).

\section{La restauration ouvrière avec service à table}

Le St. Clair est ouvert à la clientèle uniquement pour le déjeuner, sur semaine. Le nombre de couverts est rarement supérieur à 50. Fermée durant l'été, cette PME se porte pourtant bien. Le chiffre d'affaires n'est pas conséquent, mais les charges supportées sont si minimes que le résultat permet un revenu (sous la forme de bénéfices industriels et commerciaux) confortable pour le dirigeant. Concentré sur un segment particulier de la restauration commerciale, le repas ouvrier, l'établissement se différencie par sa simplicité et des prix très attractifs.

Les coûts variables sont, comme les coûts fixes, tirés vers le bas. Le coût des matières premières (pas de pertes, nombre de clients réguliers, proximité du marché d'intérêt national) ne dépasse pas $27 \%$ du chiffre d'affaires hors taxes (CAHT). Les coûts fixes se résument à un employé temps complet, un loyer raisonnable et quelques éléments de charges de gestion (charges externes). Il n'y a plus d'annuité d'emprunt (charges financières et remboursement du capital) depuis longtemps. La domination sur le plan des coûts joue donc pleinement.

\section{L'hôtellerie économique}

Concentrés sur un segment particulier du secteur, celui de tous les employés amenés à se déplacer souvent, les Formule 1, $\mathrm{B} \& \mathrm{~B}$, Première Classe, Baladins ou autres Nuit d'Hôtel se différencient par la simplicité même des services proposés. La participation active du client, les supports physiques (guichet automatique 


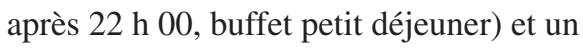
confort qualifié de «basique » sont les fondements de ces produits très fréquentés. La moyenne des frais de personnel est inférieure à $30 \%$ du CAHT, le nettoyage, dans de nombreux cas, se fait par des sous-traitants (deuxième modèle). Le coût des matières premières pour le petit déjeuner ne saurait excéder $3 \%$ du CAHT. Seuls les coûts d'occupation (leasing, loyers ou annuités selon les cas) représentent un poids important en charges fixes.

\section{Le deuxième modèle : l'avantage concurrentiel par la valeur}

La chaîne de valeur est le deuxième modèle que nous commenterons ici. La définition qu'en donne Porter est la suivante :

C'est la stratégie qui définit la manière dont est menée chaque activité et la manière dont est organisée toute la chaîne de valeur. [...] Une première distinction sommaire s'établit entre les activités principales, qui s'inscrivent dans la continuité de la production, de la commercialisation, de la distribution et de l'après-vente du produit et les activités de soutien : moyens de production, technologie, ressources humaines et autres fonctions d'ordre infrastructurel (Porter, 1993 : 43-44).
Si nous reprenons l'exemple d'EasyJet (ou Ryanair), la valeur interne créée par la firme et qui lui permet - comme certainement dans le cas des autres compagnies low cost - la mise en œuvre d'avantages concurrentiels forts, aboutit au principe suivant : le prix d'un vol augmente en fonction de son taux de remplissage et de sa popularité, jusqu'au prix maximal qui correspond à environ $50 \%$ du tarif maximal d'une compagnie traditionnelle. Cette stratégie puissante suppose une efficience de la chaîne de valeur interne. Celle-ci repose sur les éléments suivants :

- comprimer les coûts d'exploitation ;

- «no frills» (ou pas de « chichi »).

$\mathrm{Au}$ titre des coûts d'exploitation et sous un angle endogène, les low cost exercent à partir d'aéroports dits désormais de proximité, peu onéreux et très accessibles. Les institutionnels d'ailleurs contribuent très largement à l'allègement des coûts (redevances aéroportuaires attractives, campagnes de «pub destination » ou de communication ${ }^{8}$ ), récupérant leurs investissements avec les dépenses réalisées par les visiteurs/touristes. Cela a pour effet une diminution forte du coût passager ${ }^{9}$. Ces aéroports, trop longtemps délaissés, permettent par ailleurs des rotations rapides des working horses, que nous traduirons par chevaux de labours, que sont les Boeing 737, British Aerospace A146 ou Airbus A319.

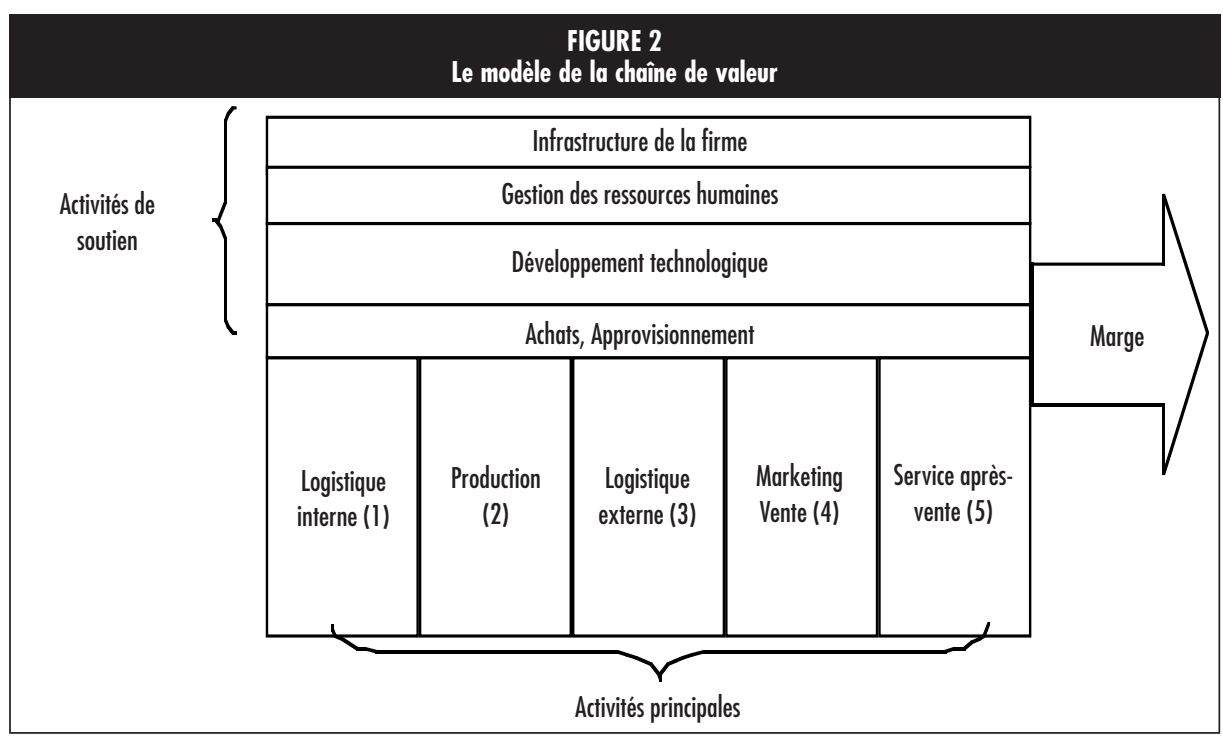

Le deuxième point clé, « no frills », s'insère parfaitement dans le modèle de la chaîne de valeur. Au-delà de l'absence de services gratuits pour les passagers (plateaux repas, journaux, boissons...), toutes les fonctions de base sont concernées.

La création de valeur résulte alors de la compression des coûts, favorisant ainsi très fortement la contribution à la marge. Les gros atouts des compagnies low cost sont sans aucun doute issus de cette approche. Nous pouvons, dans le tableau qui suit, synthétiser les incidences de cette chaîne de valeur pour les trois activités.

Tous ces éléments s'inscrivent dans la châ̂ne de valeur et, à leur niveau, participent à la réalisation des bénéfices (marge accrue).

La démonstration a été faite que les low cost sur des bases de chaînes de valeur raisonnées pouvaient être rentables et s'étendre pas à pas vers des stratégies de croissance externe (Ryanair vient d'acheter Buzz). L'absence de vision stratégique ou l'imprécision d'un axe stratégique fort alliant soit différenciation, soit concentration, soit les deux à la fois, montre les difficultés, parfois, des opérateurs à comprendre l'évolution contingente d'un secteur.

La valeur perçue, par le client, revient à l'évidence comme un concept clé de la décision de segmentation des compa- 
TABLEAU 1

Incidence de la chaîne des valeurs sur trois secteurs (transport aérien, restauration et hôtellerie)

\begin{tabular}{|l|l|l|}
\hline Transport aérien & Restauration & Hôtellerie \\
\hline $\begin{array}{l}\text { Coûts de maintenance et de } \\
\text { formation des équipages, structure } \\
\text { administrative simple (1) }\end{array}$ & $\begin{array}{l}\text { Structure simple (1) } \\
\text { Menus et prix uniques, services } \\
\text { basiques (2) }\end{array}$ & $\begin{array}{l}\text { Structure simplifiée (1) } \\
\text { Participation active des clients, nomb- } \\
\text { reux supports physiques disponibles, } \\
\text { services basiques (2) }\end{array}$ \\
$\begin{array}{l}\text { Pas de service à bord (ou payant), } \\
\text { «no frills » (2) }\end{array}$ & $\begin{array}{l}\text { Pas de promotion, de communication } \\
\text { particulière, taux de fidélité élevé (4) } \\
\text { Vente par Internet, pas de billet, } \\
\text { nettoyage en sous-traitance (3) } \\
\text { Suppression d'intermédiaires, pas des chambres, système de } \\
\text { de programme de fidélisation (4) } \\
\text { Pas de service après vente (5) }\end{array}$ & $\begin{array}{l}\text { Pente par centrale de réservation, } \\
\text { Internet, système GDS* (3) } \\
\text { Points fidélité dans certains cas (4) } \\
\text { Pas de service après vente (5) }\end{array}$ \\
\hline
\end{tabular}

Global Distribution System ou, en français, système de distribution globale.

Note: Les chiffres entre parenthèses renvoient aux chiffres du schéma sur la valeur (figure 2$)^{10}$.

gnies aériennes, mais aussi d'autres secteurs (hôtellerie avec Formule 1 en son temps, McDonald's pour la restauration commerciale) $)^{12}$.

Cette valeur est la confrontation de la valeur du produit/service en tant que tel (personnel, image), de laquelle on déduit les coûts de sa réalisation, sa fabrication.

L'optimisation des coûts par des systèmes puissants de techniques comme le yield management, la révision des principes de production du service ( no frills $»)^{13}$ en termes de logistiques interne et externe doivent, au-delà des activités de soutien (gestion de ressources humaines, R\&D, achats et approvisionnements et infrastructures de la firme), permettre de générer davantage de marge, davantage de profits.

Les hôtels à prix réduits, appelés hôtels économiques, correspondent en tous points aux deux modèles présentés ici. Dépourvus de services personnalisés ou, à l'inverse, la standardisation et la « servuction » sont poussées à l'extrême notamment sur l'axe de la participation active du client, ces établissements permettent une production du service à « bas prix ».

La démocratisation des repas, que nous avons déjà eu l'occasion d'évoquer (Callot, 1999a), sous l'impulsion des nouveaux entrants (Callot, 1997), est aujourd'hui réalité. Bon nombre de per- sonnes peuvent maintenant déjeuner à des prix raisonnables.

Les différentes formules se multiplient, notamment kiosques ou formules hors secteur (boulangeries, grande distribution), et correspondent aux fonctions liées aux repas. Celle de se nourrir (la fonction nutrition) est une des plus conséquentes en semaine, avec les déjeuners qui représentent $56 \%$ des repas pris hors domicile (Callot, 1999b). Les fastfoods, les cafés restaurants, les viennoiseries/croissanteries, les cafétérias (aussi), les restaurants routiers sont autant d'exemples qui montrent que des groupes (structurés aux ressources puissantes) ou de très petites entreprises (TPE, aux structures simples et peu fortunées) peuvent se créer un avantage concurrentiel uniquement sur ce modèle.

Nous pouvons, à partir de là, élaborer le tableau suivant qui permet, selon les secteurs, de comparer les fonctions et les bénéfices qui découlent de cette chaîne de valeur.

Les deux modèles ici adaptés à trois secteurs distincts confirment plusieurs principes managériaux qu'il importe de rappeler :

1. La nécessité de s'adapter à la contingence environnementale (nouveaux espaces, nouveaux enjeux, nouvelles technologies, nouveaux comportements, nouvelles attentes). L'Internet a, par exemple, permis d'amplifier le rayonnement des compagnies à bas coûts/prix.

2. La nécessité de s'engager dans une voie de différenciation stratégique qui permet alors de se protéger des agressions concurrentielles. La faiblesse des coûts est, par exemple, un avantage majeur qui se transforme en compétence distinctive.

3. La mise en exergue des structures et des concepts simples (sans chichi on se rappellera) qui favorisent une amplification, une contribution à la création de valeur (endogène et exogène).

\section{Conclusion}

L'hôtellerie économique, les restaurants bon marché et les compagnies aériennes low cost attestent, aujourd'hui, de l'importance de cette variable du marketingmix qu'est le prix. Les théories du marketing doivent évoluer au rythme de cette

\begin{tabular}{|c|c|c|c|}
\hline \multicolumn{4}{|c|}{$\begin{array}{c}\text { TABLEAU } 2 \\
\text { Application de la chaîne de valeur pour trois secteurs (transport aérien, restauration et hôtellerie) }\end{array}$} \\
\hline Fonctions pour le client & Secteur aérien & $\begin{array}{l}\text { Secteur de la restauration } \\
\text { (fonction nutrition) }\end{array}$ & $\begin{array}{l}\text { Secteur de l'hôtellerie } \\
\text { économique }\end{array}$ \\
\hline Fonctions principales & $\begin{array}{l}\text { Se déplacer } \\
\text { Transporter des personnes } \\
\text { d'un point à un autre sur des } \\
\text { trajets courts }<2 \text { h } 30\end{array}$ & $\begin{array}{l}\text { Se nourrir (par une démocrati- } \\
\text { sation des repas) } \\
\text { Nourrir des personnes actives } \\
\text { pour un budget indemnisé (pan- } \\
\text { ier ou indemnité forfaitaire) }\end{array}$ & $\begin{array}{l}\text { Se reposer } \\
\text { Proposer une chambre d'un con- } \\
\text { fort basique (point d'eau, couche } \\
\text { confortable) pour un prix infé- } \\
\text { rieur à l'indemnité journalière }\end{array}$ \\
\hline Bénéfices pratiques & Économie (argent et temps) & $\begin{array}{l}\text { Économie, espace décontracté, } \\
\text { rapports tribaux, garantie }\end{array}$ & Proximité des zones d'activité \\
\hline Bénéfices psychologiques & $\begin{array}{l}\text { Ego flatté (tout le monde ne } \\
\text { prend pas l'avion) }\end{array}$ & $\begin{array}{l}\text { Adhésion forte à la « tribu » } \\
\text { (ouvriers, employés, routiers) }\end{array}$ & $\begin{array}{l}\text { Garantie d'être sur place } \\
\text { le lendemain }\end{array}$ \\
\hline
\end{tabular}


contingence sociologique, technologique, économique et démographique.

Non seulement le consommateur/utilisateur/client est zappeur et infidèle, mais son degré d'exigence de confort, et donc de prestations, peut varier selon son statut et cela dans la même semaine. La contraction des frais professionnels (actifs en semaine) et la multiplication des courts séjours (non actifs en week-end) sont quelques-uns des facteurs qui favorisent une telle recherche des prix bas. La démocratisation des secteurs montre l'évolution de nos sociétés de consommation. Les budgets des ménages se contractent sur certains postes (alimentation par exemple) au profit d'autres (loisirs, santé). La gestion des coûts répercutée en bénéfice pratique pour le consommateur est le levier d'efficience pour l'entreprise.

La concurrence aussi ouverte que mondiale continue. Le couple prix/service autour des deux modèles porteriens s'impose plus que jamais. La compression des coûts au titre des activités principales et la valeur perçue alors par le client (l'avantage fort qu'il a à utiliser ce service plutôt qu'un autre) sont les voies aussi dynamiques que stratégiques que la firme doit savoir emprunter.

En ce sens, la conquête de nouveaux segments de consommateurs paraît loin d'être achevée.

Philippe Callot, docteur ès sciences de gestion (IAE et Université de Poithiers), est responsable du département Marketing/ Vente et responsable de la filière Marketing et Management des services au Groupe ESCEM de Tours.

Cet article a été obtenu grâce à la collaboration de SARL ÉTÉ, éditeur de la revue Espaces et des Cahiers Espaces.

\section{Notes}

1 À l'époque l'argument de Philip Kotler était le suivant : "Les méthodes d'établissement des prix en fonction de la demande s'intéressent [...] à l'intensité de la demande. Un prix élevé est exigé lorsque la demande est intense, et un prix faible est exigé aux périodes ou aux endroits où la demande est faible, même si les coûts unitaires sont les mêmes dans les deux cas » (1971: 428).

2 CA en hausse de $36 \%$ pour EasyJet à 486 millions de livres (729 millions d'euros environ) et 57 millions (85,5 millions d'euros environ) de résultat d'exploitation, soit + $49 \%$ en 2001 et 2002 (Source EasyJet Thevenaz, 2001), ou encore un bénéfice net de 150,9 millions d'euros au premier semestre 2002 en hausse de $71 \%$ par rapport à 2001 pour Ryanair (Coulon, 2002 : 18).

3 Après tout des chaînes comme Formule 1 affichent des T.O. (taux d'occupation qui correspondent à : n chambres louées $/ \mathrm{n}$ chambres louables x 100) généreux de $80 \%$ environ en France.

4 Directeur général d'EasyJet Suisse (Thevenaz, 2001 : 13-16).

5 Voir également le numéro spécial « $\mathrm{La}$ révolution des compagnies low cost », Espaces $\mathrm{n}^{\circ}$ 201, février 2003.

6 Observatoire Économique de Touraine, juillet-août 2002 (base $100=381$ questionnaires).

7 On sait depuis peu que la compagnie a décidé d'acheter 120 airbus A319 avec une option sur 120 autres « à prix garanti » (« Airbus s'ouvre le créneau des 'low cost' avec EasyJet », 2002 : 13).

8 Lire «Sur les ailes de low cost », $2002: 51$.

9 Coûts par passager de 67 euros pour les low cost et de 137 euros pour les compagnies classiques, source Lehman Brothers in Les Échos, 9 septembre 200 2, p. 32.

10 Soulignons que cette liste n'est pas exhaustive !

11 «L'escadrille des incompétents », $2003: 4$

12 Même si la firme, avec 30000 restaurants dans le monde, connaît une chute de son bénéfice de $45 \%$ à $893 \mathrm{M} \$$ (pour 1636 en 2001) et est en recherche de diversification (Chipotle, Donatos Pizzeria, Boston Market, Prêt-à-manger)

13 Quelles sont les véritables attentes des clients ? Les bénéfices attendus?

\section{Bibliographie}

« Airbus s'ouvre le créneau des 'low cost' avec EasyJet », Les Échos, n' 18761,15 octobre 2002, p. 13.

"L'escadrille des incompétents », Libération, 7 février 2003, p. 4.
« La révolution des compagnies low cost », Espaces $n^{\circ} 201$, février 2003.

«Sur les ailes des low cost», Les Échos, $\mathrm{n}^{\circ} 18772,30$ octobre 2002, p. 51.

Balfet, M. (2001), Marketing des services touristiques et hôteliers, spécificités, méthodes et techniques, Éditions Ellipses.

Callot, P. (1997), « Peut-on exporter la restauration commerciale française ? " Espaces $\mathrm{n}^{\circ} 148$, p. 50-55.

Callot, P. (1999a), « La restauration d'hier à demain » Espaces n ${ }^{\circ} 166$, p. 34-39.

Callot, P. (1999b), « Tous les repas ne se valent pas », Espaces n ${ }^{\circ} 161$, p. 37-39.

Callot, P. (2002), « Marketing des services : une construction sur les incertitudes de l'avenir », Market Management, Eska Editions, p. 67-78.

Capiez, A., E. Stevens, et P. Legoherel (2002), «Yield Management : Des principes à la mise en œuvre dans les activités de services », $L a$ Revue des Sciences de Gestion, Direction et Gestion, no 196-197, p. 91-104.

Coulon, Samuel (2002), « Les compagnies à bas coût veulent imposer leur modèle dans le ciel européen », Les Echos, 5 novembre, p. 18.

Dubois, P.L., et M.C. Frendo (1995), « Yield Management et Marketing des Services », Décisions Marketing, n 4, janvier-avril.

Kotler, P. (1971), Marketing Management : analyse, planification et contrôle, PubliUnion, $1^{\text {re }}$ édition.

Lovelock, C., et D. Lapert (1999), Marketing des services, Stratégie, Outils, Management, Publi-Union.

Mahe de Bosilandelle, H. (1998), Dictionnaire de gestion, vocabulaire, concepts et outils, Economica.

Porter, M.E. (1982), Choix stratégiques et concurrence, Economica.

Porter, M.E. (1985), Competitive Advantage: Creating and Sustaining Superior Performance, New York, The Free Press.

Porter, M.E. (1993), «L'avantage concurrentiel des nations », InterÉditions.

Porter, M.E. (2001), " Strategy and the Internet », Harvard Business Review, p. 63-78.

Thevenaz, J.M. (2001), L'entrée réussie dans un marché oligopolistique et contesté, Synthèse du $4^{\mathrm{e}}$ Symposium des Alpes Latines, Martigny, 9 novembre, p. 13-16.

Young, S., et B., Feigin (1975), « Using the Benefit Chain for Improved Strategy Formulation », Journal of Marketing, 72-74. 